\title{
DISCREPÂNCIAS ENTRE OS REGISTROS DE PRONTUÁRIOS ACERCA DA FARMACOTERAPIA DE PACIENTES PEDIÁTRICOS COM FIBROSE CÍSTICA
}

\author{
Pharmacotherapy discrepancies In MEDICAL RECORDS of \\ PEDIATRIC PATIENTS WITH CYSTIC FIBROSIS
}

\author{
Mônica Lopes Tonello ${ }^{1}$, Stella Pegoraro Alves², \\ Márcia de Azevedo Frank ${ }^{3}$, Denise Bueno²
}

\begin{abstract}
RESUMO
Introdução: Discrepâncias nos registros eletrônicos de medicamentos são frequentes e podem levar a erros relacionados a medicamentos. O objetivo deste estudo foi analisar as discrepâncias entre os registros médicos e farmacêuticos da terapia medicamentosa de pacientes com fibrose cística de um ambulatório multidisciplinar de pneumologia infantil do Sul do Brasil
\end{abstract}

Métodos: Estudo transversal com coleta de dados retrospectiva através de prontuário eletrônico de pacientes que foram atendidos no ambulatório de um centro de referência para Fibrose Cística entre maio e junho de 2016. Os registros médicos e farmacêuticos foram comparados e as discrepâncias quantificadas e classificadas.

Resultados: Foram analisados 81 registros de consultas ambulatoriais de 76 pacientes. Oitenta medicamentos constavam nos registros, sendo os mais frequentes polivitamínicos, enzimas pancreáticas, dornase alfa, ácido ursodesoxicólico e solução salina hipertônica. Foram identificadas discrepâncias em 96\% dos registros, totalizando 245 discrepâncias, entre elas omissões de medicamentos no registro farmacêutico (102) e no médico (80), além de doses (41) e regimes terapêuticos (23) diferentes. Foram observadas omissões de dose no registro farmacêutico (63) e no médico (52) e omissões de regime (11) em ambos.

Conclusão: A elevada taxa de discrepâncias corrobora com os resultados de diversos estudos. A conciliação tem o objetivo de promover o uso racional e diminuir os erros relacionados a medicamentos, podendo ser realizada de maneira sistematizada. A avaliação da qualidade do uso de medicamentos pode ser feita através da utilização de indicadores, sendo as discrepâncias um indicador sensível para este objetivo.

Palavras-chave: Reconciliação de medicamentos; fibrose cística; segurança do paciente; erros de medicação

\section{ABSTRACT}

Introduction: Discrepancies in electronic records of medication are frequent and may lead to drug-related errors. This study aims to analyze discrepancies between medical and pharmaceutical records of pharmacotherapy in patients with cystic fibrosis in a multidisciplinary pediatric pulmonology outpatient hospital in southern Brazil.

Methods: Transversal study with retrospective data collection through electronic medical records of patients assisted in the outpatient room of a reference center for cystic fibrosis within May and June 2016. Both records, medical and pharmaceutical, were compared and unconformities were quantified and classified.
Clin Biomed Res. 2017;37(3):181-186

1 Faculdade de Farmácia, Universidade Federal do Rio Grande do Sul (UFRGS) Porto Alegre, RS, Brasil.

2 Programa de Pós-graduação em Assistência Farmacêutica (PPGASFAR) Universidade Federal do Rio Grande do Sul (UFRGS), Porto Alegre, RS, Brasil.

3 Seção de Farmácia Clínica, Hospital de Clínicas de Porto Alegre (HCPA). Porto Alegre, RS, Brasil.

Autor correspondente:

Denise Bueno

denise.bueno@ufrgs.br

Programa de Pós-graduação em Assistência Farmacêutica (PPGASFAR), Faculdade de Farmácia, Universidade Federal do Rio Grande do Sul (UFRGS) Av. Ipiranga, 2752.

90610-000, Porto Alegre, RS, Brasil. 
Results: An amount of 81 records of 76 patients were analyzed. A total of 80 medications were observed in the records, most frequently multivitamins, pancreatic enzymes, dornase alpha, ursodesoxycholic acid and hypertonic saline solution. The discrepancies were present in $96 \%$ of records, totaling 245 discrepancies, among them omissions of medication in the pharmaceutical records (102) and in the medical records (80), doses (41) and different therapy regimes (23). We observed omissions in pharmaceutical records (63), in medical records (52) and in both (11).

Conclusion: The high level of discrepancies endorses results of a variety of studies. Reconciliation has the objective of promoting rational use and diminishing errors related to medication, when performed systematically. The evaluation of quality use of medication might be performed through the use of indicators, considering the discrepancy a sensible indicator for this goal.

Keywords: Medication reconciliation; cystic fibrosis; patient safety; medication errors

A Fibrose Cística (FC) ou mucoviscidose é uma doença genética de transmissão autossômica recessiva, caracterizada pela disfunção de uma proteína de membrana conhecida como Regulador de Condutância de Transmembrana de Fibrose Cística (CFTR). Esta proteína é responsável pelo equilíbrio entre íons e água através do epitélio, encontrada em células de diversos tecidos, como trato respiratório, glândulas submucosas, pâncreas, fígado, dutos de suor, aparelhos reprodutores, entre outros. Assim, a FC apresenta-se como uma doença multissistêmica com vários sintomas, incluindo insuficiência pancreática, alta concentração de eletrólitos no suor, e doença pulmonar obstrutiva crônica e progressiva ${ }^{1-3}$.

Segundo o Registro Brasileiro de Fibrose Cística, o país possui 3511 pacientes com diagnóstico de FC. Sul e Sudeste são as regiões com maior número de casos: 741 e 1691 pacientes, respectivamente, devido à influência da colonização europeia. O Estado do Rio Grande do Sul (RS) possui 412 pacientes acompanhados nos seus três centros de referência para a patologia 4 .

O tratamento domiciliar baseia-se no controle dos sintomas gastrointestinais e pulmonares, reposição de vitaminas deficientes e manejo das complicações ${ }^{5}$. Estudo recente realizado com pacientes infantis de um centro de referência do RS mostrou que, em média, foram utilizados 6,5 medicamentos por paciente, podendo variar entre 2 e 16 medicamentos. Os medicamentos mais utilizados foram: polivitamínicos, enzimas pancreáticas, dornase alfa, solução salina hipertônica (SSH), ácido ursodesoxicólico, antimicrobianos, entre outros. E as vias de administração mais utilizadas foram oral, inalatória, intranasal e subcutânea ${ }^{6}$.

$\mathrm{O}$ tratamento da FC envolve mudanças significativas na rotina do paciente. O tratamento é por tempo ilimitado e o número de medicamentos utilizados tende a aumentar no decorrer da vida. Estudos que visem ao melhor aproveitamento da terapia medicamentosa e ao uso racional de medicamentos são úteis para auxiliar na tomada de decisão na prática clínica dos profissionais envolvidos e para relacionar o conhecimento científico com os valores do paciente e a experiência profissional, visando à segurança e à qualidade de vida do paciente bem como à uma melhor adequação do serviço de saúde ${ }^{6-8}$.

A discrepância é definida como a ausência de um acordo entre as informações ${ }^{9}$. As discrepâncias no registro de medicamentos muitas vezes levam a erros de medicação. A inclusão do farmacêutico em um modelo interdisciplinar de conciliação de medicamentos está relacionada à redução de taxas de erro, discrepâncias e custos de atendimento, e quando associada ao uso de tecnologia, como o registro eletrônico, pode reduzir problemas relacionados à conciliação. No entanto, sabe-se que esses recursos nem sempre são suficientes para garantir a precisão da informação. Estudos são necessários para compreender as vulnerabilidades desta ferramenta, permitindo sua adaptação e melhora do serviço ${ }^{10-15}$. O objetivo deste estudo foi analisar a presença de discrepâncias entre registro médico e farmacêutico da terapia medicamentosa em um centro de referência para o tratamento da FC infantil no sul do Brasil.

\section{MÉTODOS}

Foi realizado um estudo transversal com coleta retrospectiva de dados através de um registro eletrônico. O estudo utilizou os prontuários de pacientes com FC atendidos no ambulatório de Pneumologia Infantil de um Hospital Universitário do Sul do Brasil entre maio e junho de 2016.

Foram analisados os prontuários dos pacientes de 0 a 18 anos. As discrepâncias foram quantificadas e classificadas de acordo com a Figura 1. Foram também identificados e quantificados os dados sobre a omissão da dose e/ou do regime.

Foi considerada dose diferente quando a dose do medicamento no registro médico era diferente da dose contida no registro farmacêutico. Isto é, a informação acerca do número de cápsulas/comprimidos/ampolas 
nos registros era divergente ou apresentava concentrações diferentes.

Dose omitida foi considerada quando o registro, tanto médico quanto o farmacêutico, não continha qualquer informação sobre dose, número de cápsulas/comprimidos/ampolas, volume ou concentração do fármaco. Para considerar a diferença em torno do regime terapêutico, foi feita uma comparação entre os números de administrações diárias contidas nos dois registros, ou seja, verificou-se se o esquema de administração coincidiu em ambos os registros. A omissão de um esquema foi considerada quando a falta dessa informação foi verificada.

Para a coleta e análise dos dados, foi desenvolvido um banco de dados no programa de computador Excel versão 2013. Os dados foram descritos na forma de frequências (\%) para variáveis categóricas e médias e desvio padrão para variáveis quantitativas. Este estudo foi aprovado pelo comitê de ética e pesquisa da instituição (parecer: 802.201).

\section{RESULTADOS}

Foram observados 81 registros de 81 consultas. Cinco pacientes tiveram duas consultas durante o período de análise, totalizando 76 pacientes. Quanto ao gênero, $57,9 \%$ eram do sexo feminino e $42,1 \%$ eram do sexo masculino. Em média, foram realizadas 5,4 consultas/dia. A média de idade dos pacientes foi de 7,6 anos (mediana $=6$ ), sendo que $48,6 \%$ dos pacientes eram da faixa de 0 a 6 anos de idade. Um paciente foi excluído do estudo por ter mais de 18 anos. A maioria dos pacientes era procedente do interior do RS $(47,4 \%)$, seguidos da região metropolitana $(32,9 \%)$ e da capital do Estado (13,1\%). Dois pacientes eram provenientes de outros Estados.

Foram citados nos registros 80 medicamentos diferentes, em um total de 943 vezes, sendo que $457(48,4 \%)$ deles constavam no registro farmacêutico e $486(51,5 \%)$ no registro médico. Os medicamentos mais citados foram: multivitamínicos, enzimas pancreáticas, dornase alfa, ácido ursodesoxicólico e SSH, conforme Tabela 1. A vitamina $\mathrm{K}$ injetável não foi considerada nesse estudo devido seu uso ser esporádico e ambulatorial.

Tabela 1: Frequência dos medicamentos citados ( $n=943)$.

\begin{tabular}{lc}
\hline \multicolumn{1}{c}{ Medicamento } & Frequência (\%) \\
\hline Multivitamínicos & 17,9 \\
Enzimas Pancreáticas & 16,3 \\
Solução Salina Hipertônica & 14,0 \\
Dornase Alfa & 8,4 \\
Ácido Ursodesoxicólico & 6,2 \\
Tobramicina & 5,1 \\
Budesonida & 3,4 \\
Polimixina E & 3,1 \\
Azitromicina & 2,9 \\
Fenoterol & 2,6 \\
Montelucaste de sódio & 2,1 \\
Salbutamol & 1,6 \\
Sulfato Ferroso & 1,3 \\
Omeprazol & 1,2 \\
Outros* & 13,9
\end{tabular}

*Medicamentos com frequência menor que 0,8\%.

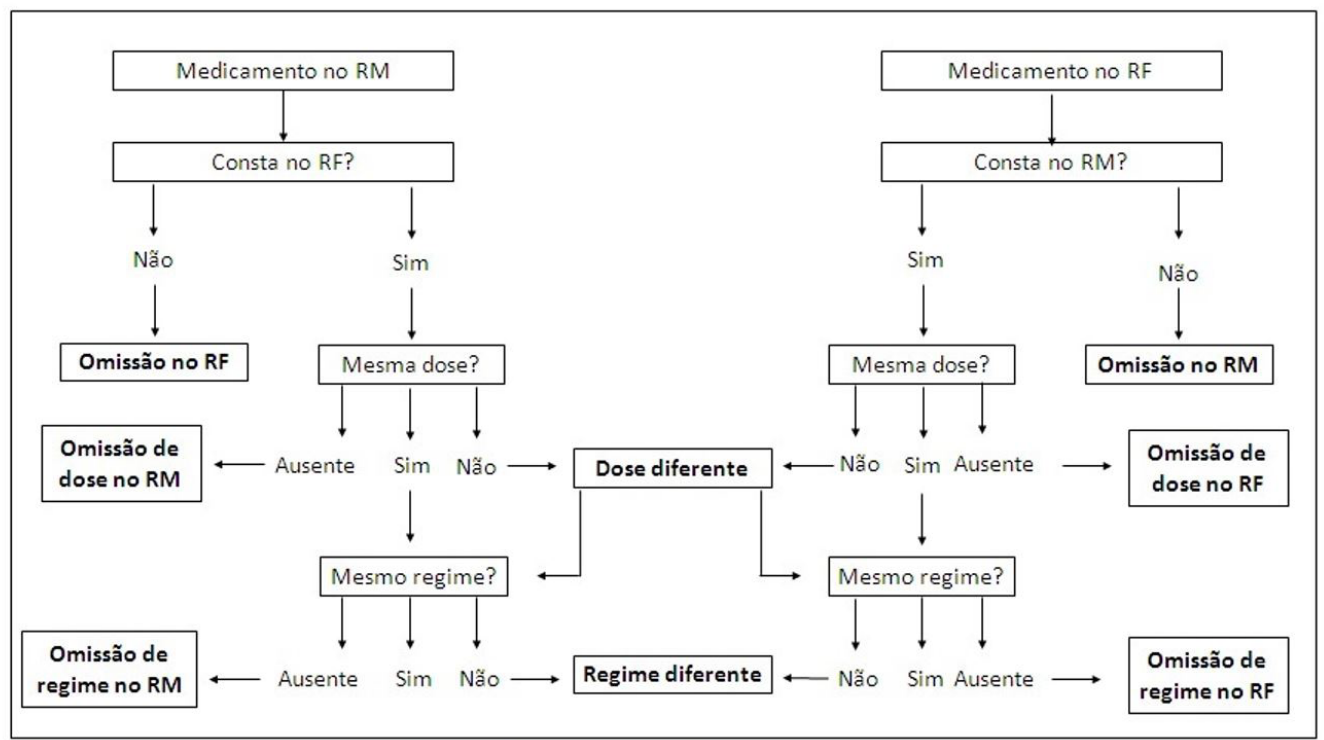

Figura 1: Fluxograma de classificação das discrepâncias dos registros de farmacoterapia. RM: Registro Médico; RF: Registro Farmacêutico. 


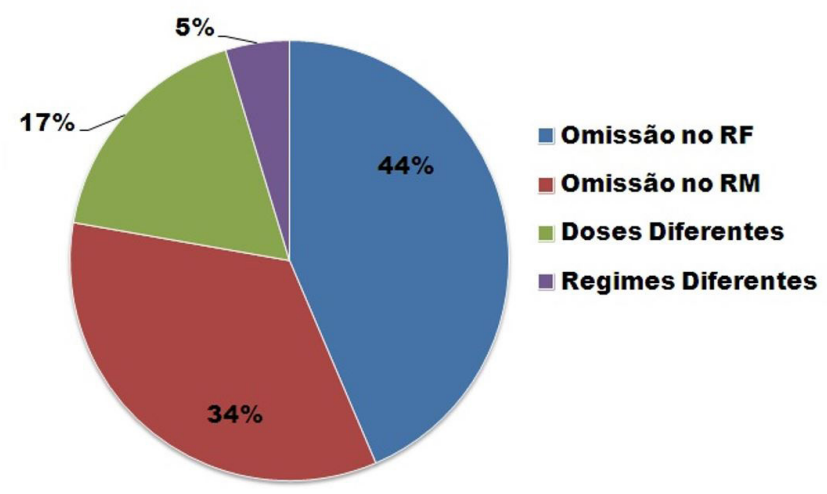

Figura 2: Tipos e frequências das discrepâncias encontradas. RF: Registro farmacêutico; RM: Registro médico.

Entre os registros analisados, $96,3 \%$ continham no mínimo uma discrepância. As discrepâncias encontradas totalizaram 245. Os tipos de discrepâncias foram apresentados na Figura 2, juntamente com a frequência com que foram encontradas. O medicamento que apresentou mais diferença entre as doses foi a enzima pancreática $(39,0 \%)$ enquanto que a SSH aparece com mais regimes divergentes $(17,0 \%)$.

Durante a coleta de dados, observou-se a falta de informações sobre a terapia medicamentosa em alguns registros, como dose e regime terapêutico. Foram quantificadas 53 omissões no registro médico e 63 omissões no registro farmacêutico.

As omissões do regime totalizaram 11 em ambos os registros. Os fármacos mais frequentemente envolvidos com a omissão de dose em ambos os registros foram SSH $(39,6 \%)$ e polivitamínicos $(29,3 \%)$. Os fármacos mais frequentemente envolvidos com a omissão do regime foram SSH (18,2\%), ranitidina $(13,6 \%)$ e salbutamol $(9,1 \%)$.

\section{DISCUSSÃO}

Estudo recente realizado no mesmo centro de referência encontrou diferenças no perfil etário (mediana de 9,7 anos) dos pacientes atendidos no mesmo ambulatório. Porém, obteve resultados semelhantes quanto ao gênero e procedência desses pacientes ${ }^{6}$.

Stewart e Lynch realizaram estudo semelhante em um ambiente de atenção primária nos Estados Unidos e encontraram frequência semelhante de discrepância $(97,5 \%)$ entre os 40 pacientes que participaram da análise. A omissão no registro médico foi o tipo mais prevalente de discrepância antes das intervenções farmacêuticas realizadas. Após as intervenções, o tipo mais frequente de discrepância foi a omissão no prontuário ${ }^{10}$. Opostamente, o presente estudo observou uma maior taxa de omissões no registro farmacêutico.

As enzimas pancreáticas apresentaram-se como o medicamento mais relacionado com as discrepâncias entre as doses, provavelmente pela necessidade da sua dose ser frequentemente alterada pelo médico, nutricionista ou até mesmo pelo paciente, dependendo dos seus sintomas.

O presente estudo apresenta frequência de medicamentos semelhante ao estudo de Alves e Bueno, os quais observaram que, segundo classificação da Anatomical Therapeutical Chemical Classification (ATC/DDD Index), os medicamentos do trato alimentar e metabolismo (enzimas pancreáticas e vitaminas) corresponderam a $45,8 \%$ do total de medicamentos prescritos. Seguidos por medicamentos do sistema respiratório ( $\mathrm{SSH}$, dornase alfa e broncodilatadores) e anti-infecciosos (antimicrobianos) com frequência de $35,4 \%$ e $14,5 \%$, respectivamente ${ }^{6}$. Nesse mesmo estudo, observou-se que $52,7 \%$ dos medicamentos prescritos eram de via de administração oral; $39,9 \%$ medicamentos de uso inalatório e $4,1 \%$ medicamentos intranasais ${ }^{6}$.

A SSH foi o medicamento com maior taxa de omissão de dose, omissão de regime e regime divergente. Estes dados ilustram a dificuldade do paciente em compreender sobre sua utilização e preparo. Uma vez que o paciente recebe a ampola de cloreto de sódio na concentração de $20 \%$ e necessita reajustá-la para $2 \%, 3 \%, 5 \%$ ou $7 \%$, dependendo do que foi prescrito e da tolerância do paciente. $O$ fato da solução ser preparada em domicílio, levando-se em conta qual foi o entendimento de quem prepara a SSH e a possibilidade de prescrição de várias concentrações, pode contribuir para as discrepâncias nos registros.

Os polivitamínicos apresentaram-se como o segundo fármaco mais relacionado com a omissão de dose. A falta de informação sobre a dose pode 
estar relacionada ao problema de abastecimento do polivitamínico, importado e fornecido pela Secretaria Estadual de Saúde do RS, que é considerado padrão para o paciente com FC, e esta situação levou os pacientes a adquirirem outros polivitamínicos de doses e apresentações variadas.

A falta de precisão nos registros de farmacoterapia ambulatorial, quando persiste, pode contribuir para propagar as discrepâncias em outros níveis de atenção à saúde, como a admissão hospitalar, pois muitas dessas informações são transmitidas de um serviço para outro. Sem uma verificação adequada dos fármacos em uso pelo paciente há maiores chances de falhar no diagnóstico e detectar problemas relacionados com medicamentos ${ }^{16}$. Os componentes da linha de cuidado ao paciente fibrocístico incluem passagem por diversos serviços de saúde, como Atenção Básica, Atenção Domiciliar, Atenção Especializada Ambulatorial e Hospitalar, entre outros. A busca pela correta verificação das informações sobre a terapia medicamentosa é essencial e a acurácia e transportabilidade das informações sobre os medicamentos podem ser consideradas indicadores de qualidade do cuidado contínuo ao paciente ${ }^{9}$.

São necessários estudos maiores acerca da origem das discrepâncias, pois elas podem ser geradas tanto por falhas do sistema eletrônico quanto pelo próprio paciente. Falhas do sistema ocorrem quando o serviço de saúde é considerado responsável pela falta de acurácia da lista de medicamentos, por exemplo, pela inserção de um antimicrobiano na lista de medicamentos que não é retirado do registro após o uso. Falhas do paciente ocorrem devido a um fator controlado pelo paciente, pois a inserção ou exclusão de medicamentos na lista depende do relato do paciente, como por exemplo, a omissão da automedicação. As falhas do sistema de registro eletrônico podem ter como causa a omissão de informações importantes, tais como: a duração do tratamento, o registro da data final de uso do medicamento e a não atualização da lista de medicamentos em uso pelos pacientes. As falhas geradas pelo paciente são causadas em grande parte pela automedicação não relatada ao profissional de saúde, pela falta de adesão e também por falhas na transmissão de informações sobre medicamentos provenientes de outros serviços de saúde ${ }^{9}$.

O processo de trabalho pode influenciar nos resultados, visto que os pacientes deste estudo são atendidos por diversos profissionais que atuam ainda de forma nuclear, inseridos em uma equipe multidisciplinar, começando sua aproximação interprofissional de forma gradativa ${ }^{6}$.

Sabe-se que as discrepâncias podem ter influência sobre o mau uso de medicamentos, porém não é possível afirmar que estas incoerências de informações apresentam um risco potencial ao paciente. Devido à variedade de divergências e medicamentos encontrados seria necessária uma avaliação crítica de cada discrepância. O desfecho clínico pode variar dependendo do medicamento, da dose, regime e do estado geral do paciente. Erros na dosagem de vitamina $\mathrm{E}$ tem significado clínico menos significativo que um erro de dose de um anticonvulsivante, por exemplo ${ }^{10}$.

Diversos esforços têm sido feitos a fim de minimizar a prevalência de discrepâncias. Smith, Coleman e Min desenvolveram e validaram uma ferramenta que permite a sensível avaliação do cuidado contínuo ao paciente ${ }^{17}$. A Medication Discrepancy Tool (MDT) consiste em um formulário que, além de verificar as discrepâncias, classifica-as em intencional ou não-intencional, juntamente com suas causas. É possível determinar as etapas do processo que são responsáveis pela origem das discrepâncias e elaborar ações ou intervenções corretivas específicas para a raiz do problema ${ }^{9,17}$.

O presente estudo apresenta como fator limitante a falta de padronização na realização das entrevistas, como a ausência de um questionário ou protocolo de atendimento. Como já citado, a rotina de múltiplos atendimentos realizados em sequência por diversos profissionais diferentes torna a consulta exaustiva para o paciente e familiar, podendo prejudicar a qualidade das informações obtidas. A coleta de dados foi realizada por um pesquisador.

Os resultados do presente estudo evidenciam a predominância de discrepâncias nos registros de medicamentos, assim como mostram outros estudos. Com o objetivo de diminuir os erros de medicamentos e promover o uso racional, a conciliação tem papel fundamental no cuidado contínuo ao paciente e, para tanto, deve ser realizada de maneira sistematizada e contínua. A utilização de indicadores de performance é a melhor forma de avaliar a qualidade do uso de medicamentos e o cuidado contínuo do paciente. A determinação das discrepâncias entre os registros de medicamentos tem se mostrado um indicador sensível para este objetivo. São imprescindíveis a avaliação e o constante monitoramento da qualidade dos serviços de saúde visando a qualidade no uso de medicamentos e a segurança do paciente.

\section{Agradecimentos}

Nosso agradecimento à equipe multiprofissional do ambulatório de pneumologia infantil do Hospital de Clínicas de Porto Alegre.

\section{Conflito de interesse}

Os autores declaram não haver conflito de interesse. 


\section{REFERÊNCIAS}

1. Simmonds NJ. Cystic fibrosis in the 21st century. Respir Med. 2010;24:8596.

2. Firmida MC, Marques BL, Costa $\mathrm{CH}$. Fisiopatologia e manifestações clínicas da fibrose cística. Rev HUPE. 2011;10:47-58

3. Ratjen F, Döring G. Cystic fibrosis. Lancet. 2003;361(9358):681-9. PMid:12606185. http://dx.doi. org/10.1016/S0140-6736(03)12567-6.

4. Grupo Brasileiro de Estudos de Fibrose Cística (GBEFC). Registro Brasileiro de Fibrose Cística (REBRAFC). GBEFC; 2014 [citado 2017 Abr 20]. Disponível em: http:// portalgbefc.org.br/wp-content/ uploads/2016/11/Registro2014_v09. pdf

5. Smyth AR, Bell SC, Bojcin S, Bryon $M$, Duff A, Flume $P$, et al. European cystic fibrosis society standards of care: best practice guidelines. $J$ Cyst Fibros. 2014;13(Suppl 1):S2342. PMid:24856775. http://dx.doi. org/10.1016/j.jcf.2014.03.010.

6. Alves SP. Utilização de medicamentos em pacientes pediátricos ambulatoriais com fibrose cística [dissertação]. Porto Alegre (RS): Universidade Federal do Rio Grande do Sul; 2016.

7. Bento AC. Adesão ao tratamento com medicações inalatórias em crianças e adolescentes portadores de fibrose cística [dissertação]. Belo Horizonte (MG): Universidade Federal de Minas Gerais; 2010.

8. Rao D, Gilbert A, Strand LM, Cipolle RJ. Drug therapy problems found in ambulatory patient populations in Minnesota and South Australia. Pharm World Sci. 2007;29(6):64754. PMid:17450422. http://dx.doi. org/10.1007/s11096-007-9123-1.

9. Orrico KB. Sources and types of discrepancies between electronic medical records and actual outpatient medication use. J Manag Care Pharm. 2008;14(7):626-31. PMid:18774872. http://dx.doi.org/10.18553/ jmcp.2008.14.7.626.

10. Stewart AL, Lynch KJ. Medication discrepancies despite pharmacist led medication reconciliation: the challenges of maintaining an accurate medication list in primary care. Pharm Pract. 2014;12(1):360. PMid:24644518. http://dx.doi.org/10.4321/S188636552014000100004.

11. Nester TM, Hale LS. Effectiveness of a pharmacist-acquired medication history in promoting patient safety. Am J Health Syst Pharm. 2002;59(22):2221-5. PMid:12455306.

12. Varkey P, Cunningham J, O'Meara J, Bonacci R, Desai N, Sheeler R. Multidisciplinary approach to inpatient medication reconciliation in an academic setting. Am J Health
Syst Pharm. 2007;64(8):850-4 PMid:17420202. http://dx.doi. org/10.2146/ajhp060314.

13. Peyton L, Ramser K, Hamann G, Patel D, Kuhl D, Sprabery L, et al. Evaluation of medication reconciliation in an ambulatory setting before and after pharmacist intervention. $J \mathrm{Am}$ Pharm Assoc. 2003;2010(50):490-5. PMid:20621867.

14. Bassi J, Lau F, Bardal S. Use of information technology in medication reconciliation: a scoping review. Ann Pharmacother. 2010;44(5):88597. PMid:20371752. http://dx.doi. org/10.1345/aph.1M699.

15. Nemeth LS, Wessell AM. Improving medication safety in primary care using electronic health records. J Patient Saf. 2010;6(4):238-43. PMid:21500611. http://dx.doi. org/10.1097/PTS.0b013e3181fe401f.

16. Gleason KM, Groszek JM, Sullivan C, Rooney D, Barnard C, Noskin GA. Reconciliation of discrepancies in medication histories and admission orders of newly hospitalized patients. Am J Health Syst Pharm. 2004;61(16):1689-95. PMid:15540481.

17. Smith JD, Coleman EA, Min SJ. A new tool for identifying discrepancies in postacute medications for communitydwelling older adults. Am J Geriatr Pharmacother. 2004;2(2):141-7. PMid:15555490. http://dx.doi. org/10.1016/S1543-5946(04)90019-0.

Recebido: Maio 03, 2017 Aceito: Jul 19, 2017 